\title{
Study The Performance Evaluation of Radio over Optical Fiber System with Optical OFDM using Different Modulation Techniques
}

\author{
Faris Mohammed Ali ${ }^{1}$, Ahmed Ghanim Wadday ${ }^{1}$, Raad Taha Balaket ${ }^{1}$ \\ E-mail: faris@atu.edu.iq, fanmnm2018@gmail.com \\ ${ }^{1}$ Department of Communications Techniques Engineering, Engineering Technical College, Al-Furat Al-Awsat Technical \\ University, Al-Najaf 31001, Iraq.
}

\begin{abstract}
This paper illustrates that the performance evaluation of radio over optical fiber (RoF) optical link has been analyzed with optical orthogonal frequency division multiplexing technique (OOFDM) through used different modulations schemes 4 -QAM, 16 -QAM, and 64 -QAM for single-channel. The new proposed system has used a combination of OFDM-RoF architecture and compare the performance of the network for different digital modulation schemes with $15 \mathrm{Gpbs}$ data rate and $5.8 \mathrm{GHz}$ radio frequency (RF), for the optical fiber lengths (20 up to 50) $\mathrm{km}$ to compensate the dispersion and other effects of distortion of wireless networks. The performance analysis is based on the optical spectrum of the transmitted signal, constellation diagrams and symbol error for four and eight channels. We can observe that the signal doesn't have any dispersion after $20 \mathrm{~km}$ of optical fiber length for the 4-QAM with OFDM technique, as well as, the system can be done well within $50 \mathrm{~km}$ of optical fiber length. In other words, the dispersion effect on the signal after $20 \mathrm{~km}$ of fiber length is less than after $50 \mathrm{~km}$, which has distorted with a large dispersion. Therefore, the simulation results show that the 64-QAM-OFDM-RoF system gives the highest value of the symbol error. We have used Optisystem "version 14" as a simulation software package.
\end{abstract}

Keywords: Radio over Fiber (RoF), Optical Orthogonal Frequency Division Multiplexing (OOFDM), Quadrature Amplitude Modulation (QAM) and Radio Frequency (RF).

\section{Introduction}

Recently, increasing demand for broadband services since the number of a mobile- cellular telephone, as well as the broadband internet, are springing up which leads to ever-growing amounts of data traffic volumes through these services [1]. Therefore, many base stations (BS) as well as a lot of money are required to meet the demand for broadband services. Rof is the best solution to this problem because it can operate a lot of BSs connected to the central station (CS) through utilizing the optical fiber and provide a cost-effective solution.

Since the optical fiber single-mode or multimode is utilized as a transfer between the base stations and the antenna, thus, RoF systems have been widely verified because of some advantages of optical fiber, such as; low loss, transparent characteristics for the transmission of the radio signal and the large bandwidth. Through using RoF systems, different RF signals which include the cellular network service and/or the wireless signals of local area network (WLAN) can be distributed with high efficiency to the outdoor ranges or densely populated areas [2]. Thus, RoF system can be the solution for the many wireless network requirements. It is a suitable wireless technology that supplied a low-cost configuration, due to transmit the optical signals modulated to the station which carries to the optical fiber without significant losses and reach the mobile users through the transmission of radio frequency. In addition, RoF is a very attractive wireless technique, because it can be used to transmit the millimeter-wave and microwave by used optical fiber for short and long distances. The radio over fiber has represented the integration of the radio frequency and optical networks, where the radio over fiber technique has used to increase the capacity of the channel of mobility and application systems, in addition decreasing consumption energy and cost [3],[4],[5],[6],[7].

OFDM is a transmission scheme that uses multiple sub-carriers to convert serial data stream with a high rate into multiple sub-carriers parallel streams with a low data rate and thus extends the symbol duration. This is helping to remove Inter Symbol Interference (ISI) [8],[9],[10],[11]. Therefore, OFDM becomes a better modulation technique that can be chosen for wireless communication to provide large data rates with high durability for the impairments the radio channel. Advanced multilevel modulations such as QAM have been proposed for keeping the signal bandwidths as low while increases the bitrate [12]. In this paper, we use the OFDM technique combined with RoF technology and 
compare the performance of the network for different digital modulation for $15 \mathrm{Gpbs}$ data rate and $5.8 \mathrm{GHz}$ of $\mathrm{RF}$, for the (20 up to 50$) \mathrm{km}$ optical fiber lengths.

Thus, this paper is organized as follows: In Section 2, it presents system design for the OFDM-RoF system which includes the proposed simulation setup for the proposed system. Section 3 provided a discussion on the performance of the proposed system with simulation results. Section 4 presents a brief concluding remarks of the study.

\section{System Design}

This section offers the design of RoF system which uses different types of modulation schemes, such as: 4-QAM, 16-QAM and 64-QAM with OFDM technique in single-channel transmission. However, the simulation of OFDMRoF system with QAM modulation scheme is shown in Figure 1 with $15 \mathrm{~Gb} / \mathrm{s}$ data rate and $5.8 \mathrm{GHz}$ RF when the length of fiber optic is also changed from (20 up to 50$) \mathrm{km}$.

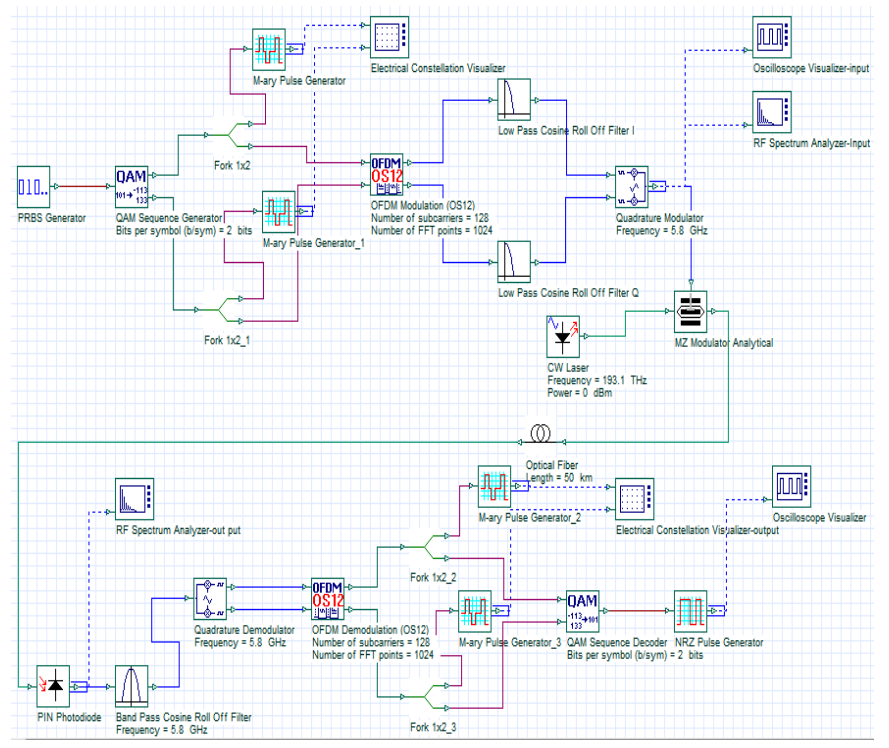

Fig. 1. Proposed simulation setup of the OFDM-RoF system model

The OFDM-RoF system consists of three main parts which are; transmitter, fiber channel and receiver. However, the transmitter part contains two main sides, such as; RF side and the optical side for generating optical signals. The "CW laser array" pumps the OC for the 8-channels at $0 \mathrm{dBm}$ power by starting from $193.1 \mathrm{THz}$ frequency with 100 $\mathrm{GHz}$ channel spacing. Also, the length of the optical fiber is changed from (20 up to 50) km, as well as the parameters of MUX and DEMUX are $100 \mathrm{THz}$ channel frequency. The number of subcarriers that are utilized of OFDM technique in this system are 128 and 1024.

However, the data rate at the transmitter side for the proposed system model is encoded first, after that, it is converted into a constellation map of the used QAM scheme. This data is interpreted in the OFDM system as a frequency-domain data, then these frequency-domain data has converted to a time-domain signal by the used of the IFFT technique of the OFDM block. Furthermore, the output of the IFFT signals have transmitted to the channel after adding cyclic prefix (CP) to combat multipath by making channel estimation easy, then used digital to analog converter D/A to carry the OFDM time signals to the analog appropriate form. Finally, the modulated laser diode has used to create optical signals which pass by the optical link to the wireless channel. An external modulator MZ Modulator analytical is placed between the CW laser and the radio frequency RF modulated signal because of high modulation efficiency. The receiver total noise and PIN photodiode are calculated and overlaying it on an ideal photodiode signal current.

At the receiver side, the reverse process is done after the detector the optical signal PIN photodetector. However, after receiving the signal will be converted to the frequency domain through used FFT operation after the CP. Finally, the received data will back from the optical link in OFDM demodulation or receiver part of OFDM.

\section{Results and Discussions}

In this section, the simulation results of the signal-channel of the OFDM-RoF system have conducted for different QAM schemes. However, in this simulation, QAM signal which uses $(4,16$ and 64$)$ bits per symbol is used. The number of bits in each symbol will $(2,4$ and 6$)$ and the constellation diagram result is given by using the formula $2 \mathrm{n}$. 
However, this study assumes that all simulations parameters setting for the proposed system are available and fully known, while the rest will be assigned to the default value of the optical system software. The performance of RoF optical link has been analyzed with the optical OFDM technique through used different modulations schemes for single-channel. The proposed system uses the combination of OFDM-RoF architecture and compares the performance of the network for different digital modulation schemes with $15 \mathrm{Gpbs}$ data rate and $5.8 \mathrm{GHz}$ radio frequency (RF), for the optical fiber lengths (20 up to 50$) \mathrm{km}$.

The results of the radio frequency spectrum of the transmitted signals and received signals for the Radio over Fiber-OFDM system for different QAM schemes after $20 \mathrm{~km}$ with more harmonics at the sideband of the spectrum as shown in Figure 2. The spectrum of RF is for four data streams, where the four data streams of bit rate 5Gbps are modulated into four RF subcarriers of frequencies $(2.4,5.8,10$ and 15) $\mathrm{GHz}$ which are multiplexed in RF domain. The architectures block system of a transmitter side with an actual signal has proposed in this paper for causing the radio frequency OFDM signal to be a complex moderate frequency in order to suppress one of the sidebands with an optical filter. Furthermore, at the reception side, the photodiode is used to detect the optical signal and then demodulated it. In addition, to remove the single-sideband, the RF frequency must be chosen in this work.

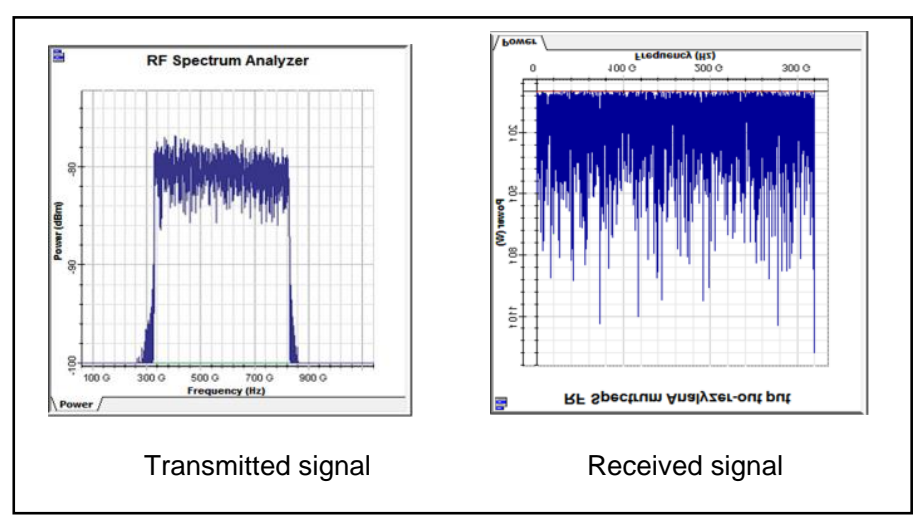

A- 4 QAM Scheme



C- 64 QAM Scheme

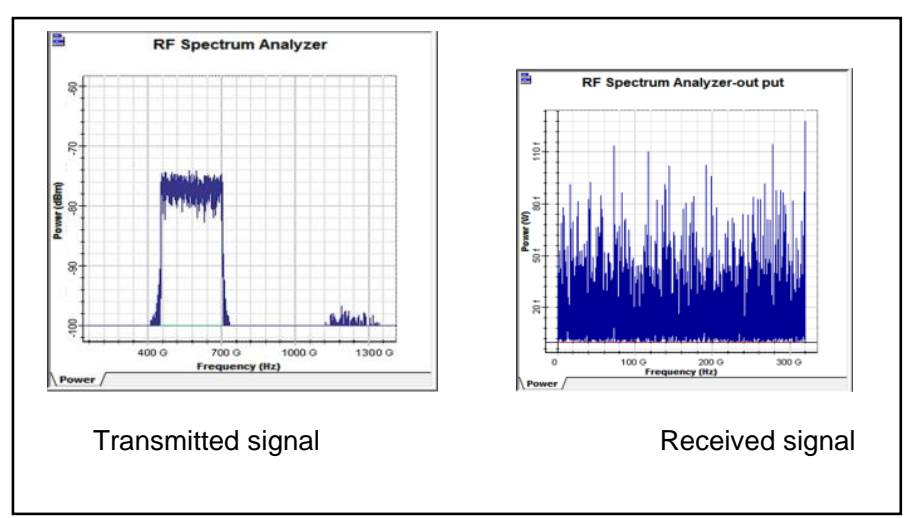

B-16 QAM Scheme

Fig. 2. The Radio Frequency spectrum of the transmitted signals and received signals for RoF-OFDM system for different QAM schemes $(\mathrm{A}, \mathrm{B}, \mathrm{C})$

Figure 3 shows the constellation diagram of the $4 \times 15$ Gbps for the 4-QAM-OFDM-RoF system after (20 and 50) $\mathrm{km}$ of fiber optic length. However, the constellations diagram of the transmitted signals is taken from the output of the M-ary pulse generator. However, it is can be observed that the signal doesn't have any dispersion after $20 \mathrm{~km}$ of optical fiber length for the 4-QAM with OFDM technique. As well as, the system can be done well within $50 \mathrm{~km}$ of optical fiber length. In other words, the dispersion effect on the signal after $20 \mathrm{~km}$ of fiber length is less than after 50 $\mathrm{km}$ which has distorted with a large dispersion. However, when the OFDM technique is used, the signal can be detected after $45 \mathrm{~km}$ of fiber without dispersion, after that the dispersion impact begins on the signal. 


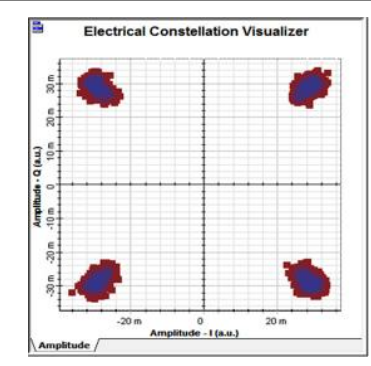

After $20 \mathrm{~km}$ length of optical fiber

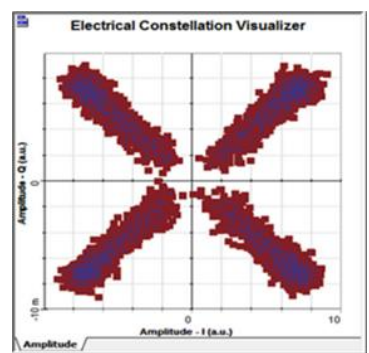

After $50 \mathrm{~km}$ length of optical fiber

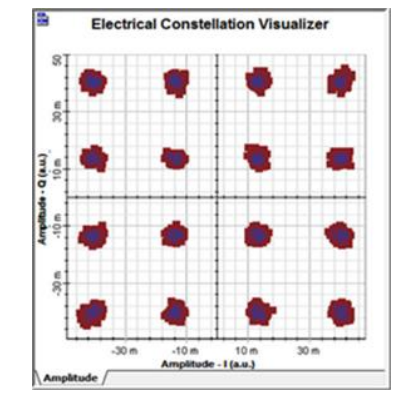

After $20 \mathrm{~km}$ length of optical fiber

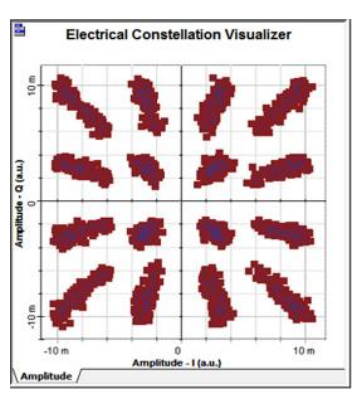

After $50 \mathrm{~km}$ length of optical fiber

A- 4 QAM

B- 16 QAM

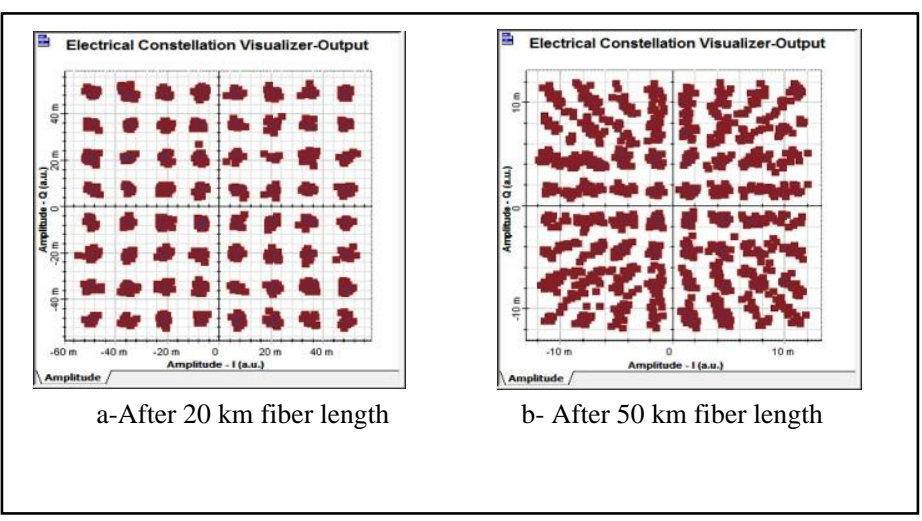

\section{C- 64 QAM}

Fig. 3. The constellation diagram of the received signal with OFDM technique for different $Q A M$ schemes $(A, B, C)$

According to Figures 2 and 3, it can be noticed that the performance of system 64-QAM with OFDM is best than 4-QAM and 16-QAM, this is because an OFDM technique fixed the problem of Inter-Symbol Interference (ISI). Also, it is noticed that the system can be done well within $50 \mathrm{~km}$ of optical fiber length. As well as, the signal doesn't have any dispersion after $20 \mathrm{~km}$ of optical fiber length for the 16-QAM with OFDM technique.

Figure 4 and 5 have shown the symbol error versus the signal to noise ratio (SNR) after 20 and $50 \mathrm{~km}$ of optical fiber length for 4-channels of RoF-OFDM system. However, from this figure, we can see that the symbol error of 4QAM-RoF-OFDM at $10 \mathrm{~dB}$ SNR equal to $-8 \mathrm{~dB}$, while it is equal to $-5 \mathrm{~dB}$ and $-2.3 \mathrm{~dB}$ for the $16-\mathrm{Q} A M-\mathrm{RoF}-\mathrm{OFDM}$ and 64-QAM-RoF-OFDM, respectively, at the same SNR.



Fig. 4. The symbols error versus the SNR after $20 \mathrm{~km}$ optical fiber length 


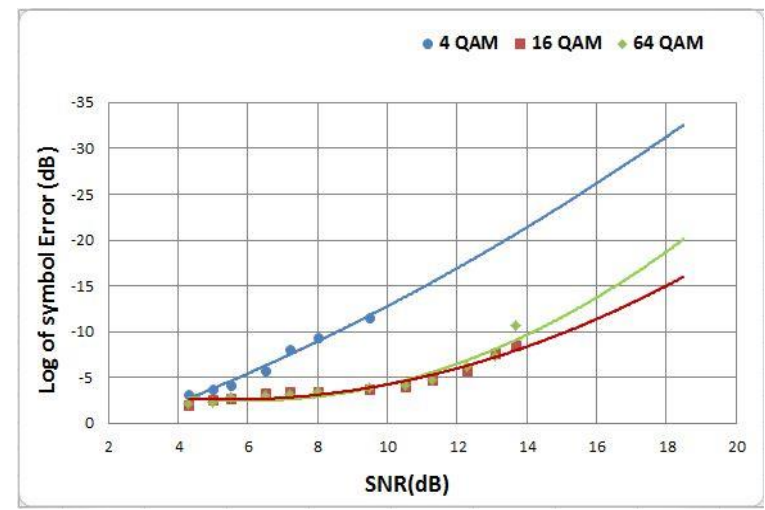

Fig. 5. The symbols error versus the SNR after $50 \mathrm{~km}$ optical fiber length

\section{Conclusions}

In this paper, we used of OFDM technique in the optical access networks communication system through combining the modulation OFDM with the Radio over Fiber system to create a high-efficiency communications system that uses the advantages of optical fiber with very high efficiency with effectively uses the bandwidth. The proposed system has been performed through using three different modulation schemes for a single-channel, such as; 4-QAM, 16QAM, and 64-QAM with data rate $15 \mathrm{Gpbs}$ and radio frequency $5.8 \mathrm{GHz}$, for the optical fiber lengths (20 up to 50) $\mathrm{km}$. The simulation results show that the 64-QAM-OFDM-RoF system gives the highest value of the symbol error and a slight difference of symbol error between 4-QAM-OFDM- RoF and 16-QAM-OFDM- RoF. Thus, we can conclude that the 64-QAM-OFDM-RoF system gives acceptable performance and reduces the complexity of the system that uses a high level of modulation schemes. In addition, with the increase of data, began the output waveforms of the RF spectrum analyzer to broaden with the decrease the quality. Furthermore, the constellations diagram output also shows increases in the constellation points. Thus, the OFDM-ROF system has possessed a better efficiency comparing with the existing communication standards. 


\section{References}

[1] A. K. Vyas, and N. Agrawal,: Radio over fiber: future technology of communication. International Journal of Emerging Trends \& Technology in Computer Science (IJETTCS). Vol. 1, No. 2, (2012).

[2] H. A. Sharshar,: Performance enhancement of radio over fiber communication systems with wavelength division multiplexing and modulation techniques. International Journal of Advanced Research in Computer Engineering \& Technology (IJARCET). Vol. 3, No. 1, (2014).

[3] Z. Kornain, M. A. Abu, and M. Y. Yacob, : The simulation of indoor service range prediction of wireless radio access point for radio over fiber system. IACSIT International Journal of Engineering and Technology. Vol. 5, No. 1, (2013).

[4] G. Aarthi, N. Sangeetha,: Comparative analysis of analog and digital radio-over-fiber systems. International Journal of Engineering Sciences \& Research Technology (IJESRT). Vol. 3, No. 2, 2014.

[5] G. de Valicourt, A. Nkansah, D. Wake,: Performance comparison of single and two-section RSOAs in radio-overfiber links. IEEE Photonica Technology Letters. Vol. 25, No. 2, (2013).

[6] B. R. Ballal and S. Nema,: Performance Comparison of Analoge and Digital Radio over Fiber link. International Journal of Computer Science \& Engineering Technology (IJCSET), Vol. 3, No. 6, (2012).

[7] H. Zhou, S. Mao and P. Agrawal,: Optical power allocation for adaptive transmission in wavelength-division multiplexing free space optical networks. Digital Communication Networks. Science Direct. Vol. 2015, No. 1, pp. 171-180, (2015).

[8] W. K. Saad, M. Ismail and R. Nordin,: Survey of Adaptive Modulation Schemes in MIMO Transmission. Journal of Communications (JCM), Vol.7, No. 12, pp. 873-884, (2012).

[9] Y.-K. Wong, S. M. Idrus, and I. A. Ghani,: Performance Analysis of the OFDM Scheme for Wireless over Fiber Communication Link. International Journal of Computer Theory and Engineering, Vol. 4, No. 5, (2012).

[10] S. Mahajan, N. Kumar, : Performance Analysis of Coded OFDM Signal for Radio over Fiber Transmission. IOSR Journal of Electrical and Electronics Engineering (IOSR-JEEE). Vol. 1, No. 1, pp. 49-52, (2012).

[11][11] Y.-M. Lin,: Next-Generation OFDMA-Based Passive Optical Network Architecture Supporting Radio-overFiber. IEEE Journal on selected areas in communications. Vol. 28, No. 6, (2010).

[12] W. K. Saad, M. Ismail, R. Nordin and I. Shayea,: Throughput Performance of Adaptive Modulation and Coding Scheme with Link Adaptation for MIMO-WIMAX Downlink Transmission. Journal of Asian scientific research. Vol.2, No. 11 , pp. 641-650, (2012). 